Which brings me to the Chief Examiner's response to the letter (Psychiatric Bulletin, March $1994,18,175)$. However necessary the exam, as a threshold and a stimulus, it can also impede one's training. As a registrar one usually rotates through six month slots of psychiatric subspecialties. When the candidate sits the examination in one such period, with time off for a revision course and independent study leave, it is unlikely that he or she will have the energy or motivation to read up about the subspecialty he or she is attached to. With a pass rate of 195 out of 405 candidates this is likely to happen more than once.

Maybe registrar training could be organised like GP training; for example, a rotation of two years through different attachments like general psychiatry, child and family therapy, old age psychiatry, community psychiatry, learning disability and forensic psychiatry. Each could be examined in their own right, and date. This would enable registrars to study the subject they are working in and leave enough time for a three year higher psychiatric training and thus comply with 'specialist-training'.

R. STOCKING KORZEN, Hillview Lodge, Royal United Hospital, Combe Park, Bath BA1 3NG

Sir: I read with interest the comments by Akintunde Akinhunmi pertaining to the MRCPsych Part II examination (Psychiatric Bulletin, March 1994, 18, 175).

The College rightly attaches the utmost importance to the clinical component of both examinations (Part I and Part II) leading to Membership. Candidates cannot pass unless the clinical is successfully negotiated. Perhaps it would therefore be more appropriate to exclude from the written papers candidates who fail the clinical. In its current form I believe candidates should not be excluded from the clinicals if they have already failed the written papers; in any case, I doubt if there would be adequate time to mark the written papers before the clinicals in the case of Part II. A further consideration are the criteria which need to be met for success in the examination. Currently a fallure in the written papers does not mean automatic fallure overall, providing the candidate passes the clinical; I believe it should stay that way.

I can understand the anxieties about the cost of the examination. The College has a duty to minimise these, while maintaining standards. Perhaps the activities of the examinations department could be audited and the results published annually in the Bulletin?

Performance in the clinical examination might actually be made worse by knowledge of success in the written papers (leading to heightened anxiety)!
Finally, I do not think it would be fair on candidates who are borderline if those who have clearly passed know their results first. The only way to speed up the processing of results would be to employ more staff - which would increase costs. I feel strongly that candidates should not be informed immediately if successful. There should be opportunity for reflection by the Examination Sub-Committee. For those candidates who have failed the examination, feedback on performance should be prioritised; some candidates have been receiving their feedback only days or weeks before their next attempt. This is clearly unsatisfactory.

STEPHEN M. JoNes, Norwich Psychiatry Rotation, West Norwich Hospital, Norwich NR2 3TU

Sir: I note the points that Dr Jones makes and will make sure that these, together with other points made regarding the examination, are brought to the attention of the committee reviewing the examination.

Sheila Mann, Chief Examiner, The Royal College of Psychiatrists

\section{Mental Health Act (MHA) as an exam topic for the MRCPsych?}

Sir: The issue of the need for training in the MHA arose from the recent Mental Health Act (MHA) Conference in London. Indeed, section 12 approval of psychiatrists does not include formal testing in the MHA. How better to encourage trainees to learn the MHA than to make it an examinable topic? The difficulty, as I understand, lies in the difference between Scottish, Irish, English/Welsh laws, and that there are candidates from Hong Kong.

I put the issue to my colleagues in the St George's Hospital Psychiatric Rotations (South West Thames Region). Fifty questionnaires were distributed to senior house officers and registrars and 40 responded; 11 had no Part I, 26 had Part I and 3 had Part II. Thirty-four were keen to have formal teaching in the MHA. Twenty-six (65\%) rated their knowledge of the MHA as fair. 11 as 'poor' and one said he/she knew nothing! The most common source of knowledge was 'onthe-job' (93\%) but $60 \%$ also who read up on the MHA. Among other sources of knowledge, one trainee included 'social worker', and another said 'lawyer'!

Twenty-eight (70\%) wanted the MHA to be an examinable topic in the MRCPsych, while only nine said no, and three said they did not know. It was clear that the majority were recognising the importance of the MHA although, in this group of 28 trainees, six $(21 \%)$ rated their knowledge of the MHA as poor. 